\section{DANISH WORK WITH PAIN AND THE NEONATE, INCLUDING A NATIONAL GUIDELINE}

\section{H. Haslund}

\section{Aalborg Hospital, Aarhus University Hospiotal, Aalborg, Denmark}

As Special Interest Group of neonatal nursing we have been working for several years with the prevention and mangement of pain in the newborn at a national level. We have arranged seminars with focus on the latest evidence on the topic. We have discussed implementation of nurse practice that matches international resommendations in the group but also amon collegues at semninars and in our own neonatal units. Autumm 2007 we conducted a national questionarie survey, idetifying problem areas that needed special attention. We fhere ound out that pain score scales was poorly implemented.

In order to facilitate the acces to relevant knowledge concerning pain and neonates including the use of pain scores we decided to make a national guideline. Alitterature study was conducted and latest evidense thus included in the guideline. In the guideline we chose to explicitate the use of pain score scale and the relevant use of non farmacological interventions including an integration of NIDCAP principles.

Concluding: This proces of working with improving qualilty of nurse practice regarding pain management.has made it clear to us, that it is important that a group of nurses work probably years with the subject in order to ensure that teaching and discussion as well as guidelines are available as tools in the clinical proces. As inspiration we hereby want to share the proces of this work as well as the actual clinical national guideline of pain and the neonate in Denmark.

\section{MANAGEMENT OF CURRENT PRACTICE FOR PAIN PREVENTION IN MINOR PROCEDURES IN THE NURSERIES: A REGIONAL INVESTIGATION IN LOMBARDIA.}

\author{
A. Battan, C. Farina \\ Neonatology, MBBM Ospedale S. Gerardo, \\ Monza, Italy
}

Background and aims: Pain experience can alter clinical outcome, brain development, and subsequent behaviour in newborns. The aims of this study were to investigate pain management, nonpharmacological treatment and use of pain scores during minor painful procedures, in healthy and low risk newborns, admitted in nurseries.

Methods: In the 2008 a questionnaire was sent to the 68 nurseries, of the whole Region.

Results: The rate of response to the questionnaire was $70,6 \%$. Of all the Hospitals, $75 \%$ had pain prevention educational programs and $43 \%$ oriented on nonpharmacological treatment. Guidelines were available in $56 \%$ of the nurseries, validated pain scores were used by $35,4 \%$ of the units and where adopted, NIPS, were used in $20,8 \%$. Further observations: the mean number of procedure was $3,7 /$ newborn; pain prevention ranged between $23 \%$ to $40 \%$ for each procedure. Education was related to the level of care units, more planed in the II and III then in the I level (59\%-53\% vs $19 \% ; p=0,045)$. Moreover, a specific educational program resulted in a greater use of guidelines $(63 \%-19 \% ; p=0,006)$. Where number of neonates/year/nurses was greater, guidelines were more utilized $(193-154 ; p=0,057)$.

Conclusions: Compared with the few studies available, pain prevention was higher (max 48\%), even if applied nonsystematically, but depending on the individual attitude of caregivers. Where used, nonpharmacological treatment was according to the recommendations of the Italian Neonatal Society (2005). Breastfeeding as a preventive measure during hell-lancing was utilized in $25 \%$ units adopting prevention, whereas it is considered the most effective and simple treatment. 